Supporting Information for

\title{
How Size and Aggregation of Ice-Binding Proteins Control their Ice Nucleation Efficiency
}

\author{
Yuqing Qiu, Arpa Hudait, and Valeria Molinero* \\ Department of Chemistry, 315 South 1400 East, \\ The University of Utah, Salt Lake City, Utah 84112-0580, USA \\ *corresponding author, email: Valeria.Molinero@utah.edu
}

\section{A. Lattice mismatch to ice of the ice-binding molecules of this study.}

Table S1 shows the lattice mismatch to ice, defined as in refs. ${ }^{1-3}$, of the four sets of ice-binding molecules of this study. In the case of TmAFP, the mismatch corresponds to an average over the different pairs of neighboring $\mathrm{OH}$ groups at the binding site. In the other ice-binding molecules, the mismatches are the same for all pairs of $\mathrm{OH}$ groups.

Table S1. Average lattice mismatch of the hydroxyl groups of the IBS with respect to the positions of water molecules in the basal plane of ice.

\begin{tabular}{|c|c|c|}
\hline Protein & $\delta a(\%)$ & $\delta b(\%)$ \\
\hline TmAFP & +7 & -7 \\
\hline TmINP & +7 & -9 \\
\hline PsINP & 0 & -7 \\
\hline AlcohoIINP & +7 & -7 \\
\hline
\end{tabular}

\section{B. Role of the backbone and $\mathrm{OH}$ groups at the IBS on the ice nucleation efficiency of model proteins.}

To understand the gap in ice nucleation efficiency between TmAFP and TmINP with the same size of binding site, we produce two chimeric versions of ice nucleating proteins that allow us to evaluate the separate contributions of the chemical heterogeneity of the protein backbone and the slight positional disorder of the $\mathrm{OH}$ groups of the threonine residues of TmAFP on the low freezing efficiency of this antifreeze protein. TmAFP has two rows of threonine residues at its binding site, one has $4 \mathrm{Thr}$ and the other has 7 (Figure S1), but the closest to the C-terminus does not bind to ice. ${ }^{2}$ To make a fair comparison between TmAFP and TmINP, we build a protein that repeats the loop we use to produce TmINP (residues 27 to 38 of TmAFP) six times, preserving the average lattice mismatch to ice of TmAFP, but replacing the two Thr on two loops close to the N-terminus with Ala. We refer to this backbone as TmINP* (Figure S1). For the first chimera, we take the IBS of TmAFP and the backbone of TmINP* with six repeating loops, align the binding sites Thr65 and 
Thr63 on the first loop of TmINP* to make a chimeric INP of TmAFP IBS + TmINP* backbone. For the second chimera, we take the regular IBS of TmINP* containing ten binding sites (four in one column and six in the other) and the backbone of TmAFP, align the first row of Thr in the place of Thr65 and Thr63 TmAFP to make a chimeric INP of TmINP* backbone +TmAFP IBS. Figure S1 shows snapshots of the two chimeras, together with TmAFP and TmINP. We solvat'e each of these four ice-binding proteins in a $13 \mathrm{~nm} \times 13 \mathrm{~nm} \times 8 \mathrm{~nm}$ periodic simulation cell containing 42665 water molecules, and measure their ice freezing temperature $T_{\text {het }}$ in a cooling ramp at the rate of $1 \mathrm{~K} \mathrm{~ns}^{-1}$ in the $N p T$ ensemble at 1 bar. Table S2 reports the freezing efficiency $\Delta T_{\mathrm{f}}=T_{\text {het }}-T_{\text {hom }}$, for each of the proteins $\left(T_{\text {hom }}=202 \mathrm{~K}\right.$ at this cooling rate, $\left.{ }^{4}\right)$. The error bar of $\Delta T_{\mathrm{f}}$ is computed from five independent simulations of ice nucleation with each protein.

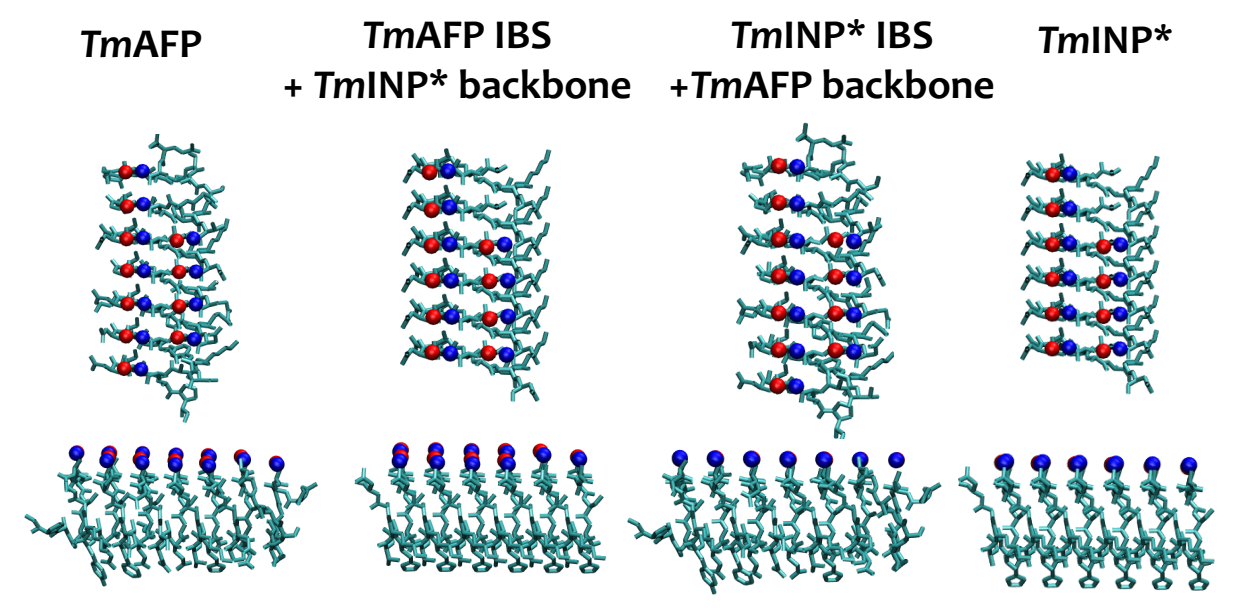

Figure S1. Snapshots of TmAFP, TmINP* and the mix-and-match chimeric INPs. Color code is the same as Figure 1. Cyan bonds represent the backbones. Red and blue balls are the methyl and hydroxyl groups of IBS. The sources of backbones and IBS are labeled.

TmAFP nucleates ice only $2 \mathrm{~K}$ above of the homogeneous limit, much lower than the TmINP* that exposed the same size of IBS. Table S2 suggests that is the synergism between the unevenness of the IBS of TmAFP and the chemical heterogeneity in the backbones of TmAFP that is responsible for the relatively low freezing efficiency of that antifreeze protein.

Table S2. The freezing efficiency $\Delta T_{\mathrm{f}}$ for the four chimeric INPs in Figure $\mathrm{S} 1$

\begin{tabular}{|c|c|c|}
\hline IBS & Backbone & $\Delta T_{\mathrm{f}}(\mathrm{K})$ \\
\hline \multicolumn{2}{|c|}{ TmAFP } & $2 \pm 1$ \\
\hline TmAFP (uneven) & TmINP (repeating 6 loops) & $8 \pm 1$ \\
\hline TmINP (even) & TmAFP (heterogeneous backbone) & $9 \pm 1$ \\
\hline \multicolumn{2}{|c|}{$T m I N P$} & $11 \pm 1$ \\
\hline
\end{tabular}

Table S2 suggests that is the synergism between the unevenness of the IBS of TmAFP and the chemical heterogeneity in the backbones of TmAFP that is responsible for the relatively low freezing 
efficiency of that antifreeze protein. The dependence of $\Delta T_{\mathrm{f}}$ on the protein backbone sequence also explains the lower ice nucleation efficiency of PsINP compared to TmINP (Figure 2), despite the better matching of PsINP to ice (Table S1). The results are consistent with those of a previous study of ice nucleation by alcohol monolayers, which demonstrated that both the positioning of the alkyl chains of the alcohols and the distribution and structural fluctuations of the $\mathrm{OH}$ groups have a large effect on the ice nucleation temperature of the monolayers. ${ }^{1}$

\section{Determination of the size of the critical ice nucleus using committor analyses.}

We compute the committor probabilities for the ice nuclei on the $L=5 \mathrm{~nm}$ long TmINP using the procedure described in Methods. To find the critical size we first compute the probability $P_{\mathrm{B}}$ that ice nuclei with $N_{\text {ice }}$ water molecules crystalize (circles in Figure S2), and then fit these points with the inverse trigonometric function, committor $P_{\mathrm{B}}=2^{*} \arctan \left(0.012^{*}\left(N_{\text {ice }}-90\right)\right) / \pi+0.5$ (red line in Figure S2). We find that the critical ice nucleus at $P_{\mathrm{B}}=0.5$ contains about 90 water molecules. Snapshots in Figure 2a are taken from configurations with an ice cluster of 86 water molecules (blue point of Figure S2).

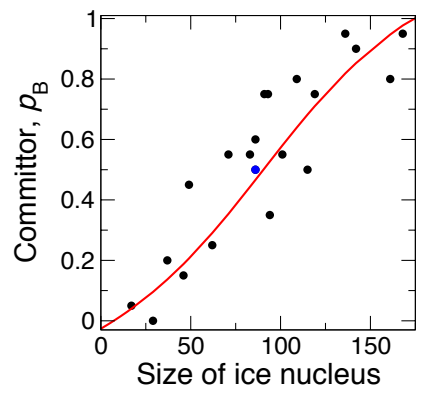

Figure S2. Committor probability $P_{\mathrm{B}}$ of the $5 \mathrm{~nm}$ long model $T m \mathrm{mIN}$ at $220 \mathrm{~K}$ increases with the size of the ice cluster. Black points are the results from the calculations and the red curve is the best fit, $P_{\mathrm{B}}$ $=2^{*} \operatorname{atan}\left(0.012^{*}\left(N_{\text {ice }}-90\right)\right) / \pi+0.5$. The blue point is the size for which the probability to commit to the liquid or ice states are identical, i.e., the critical size of the ice nucleus at $220 \mathrm{~K}$.

\section{Ice nucleation temperature vs area for strongly and weakly ice-binding surfaces.}

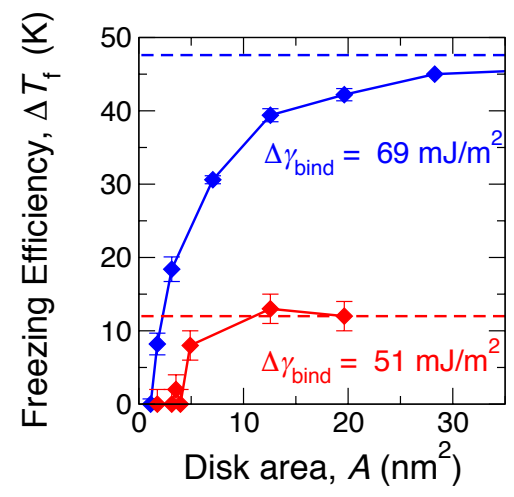

Figure S3. Freezing efficiency for disks of a strongly binding alcohol monolayer (blue diamonds) and graphite (red diamonds) as a function of the area of their ice-binding surfaces. The freezing temperatures were determined in simulations with $\mathrm{mW}$ water at a cooling rate of $1 \mathrm{~K} / \mathrm{ns}$ with the 
alcohol monolayer model of ref. ${ }^{1}$ and the graphite model of ref. ${ }^{5}$. The values of binding free energy to ice per area, $\Delta \gamma_{\text {bind, }}$, reported for each surface in the figure were determined using the procedures explained in the Appendix. A stronger binding free energy to ice results in ice nucleation efficiency for smaller surfaces and also higher efficiencies of the surfaces of unlimited sizes (shown with dashed lines in the same color as the data).

\section{E. Ice nucleation temperatures of TmAFP, PsINP, and their aggregates predicted by classical nucleation theory implemented in HINT.}

In Tables S3-36 below, $\mathrm{A}(T)$ is the pre-exponent -which includes the number of proteins- and $\Delta G^{*}(T)$ the barrier at different temperatures but same nucleation rate $\omega$, computed using CNT with the HINT algorithm. Changes in the concentration of protein are included into the pre-exponent $A(T)=A_{\mathrm{o}}(T) N / N_{\mathrm{o}}$, where $A_{\mathrm{o}}(T)$ is the value of the pre-exponent for the reference with $N_{\mathrm{o}}$ ice nucleanting particles. Our reference number $N_{o}$ of proteins per droplet is between $10^{4}$, the density of Ps. Syringae bacteria in the experiment of ref. ${ }^{6}$ from which we derive the maximum ice nucleation efficiency of these ice nucleating proteins, and $\sim 1$, the number of bacteria that and other studies estimate that reach this ice nucleation temperature per droplet. ${ }^{6-7}$

\section{E.1. Effect of concentration, aggregation, and lengthening of the binding site on ice nucleation by TmAFP}

The evolution of $T_{\text {het }}$ with number $N$ of individual TmAFP per droplet shown Table S3 indicates that the $4 \mathrm{~K}$ increase of $T_{\text {het }}$ upon 200-fold increase in concentration of TmAFP observed in the experiments of ref. ${ }^{8}$ cannot be explained by a mere increase in total nucleating area, and is mostly due to aggregation of TmAFP to more slightly more efficient ice-nucleating surfaces (see Table S4).

We model TmAFP as $1.3 \mathrm{~nm}$ wide and $2 \mathrm{~nm}$ long (4 ice-binding TxT loops), using $\tau=10 \mathrm{pN}$ and two sets of $\Delta \gamma_{\text {bind }}$ at the melting point:

i) $\Delta \gamma_{\text {bind }}=-62.6 \mathrm{~mJ} \mathrm{~m}^{-2}$ which we deduce from the experimental $T_{\text {het }}=271 \mathrm{~K}$ for Ps. Syringae (see manuscript).

ii) $\Delta \gamma_{\text {bind }}=-55.1 \mathrm{~mJ} \mathrm{~m}^{-2}$. This value is obtained by first computing $\Delta \gamma_{\text {bind }}=-61.8 \mathrm{~mJ} \mathrm{~m}^{-2}$ for TmAFP in the united atom simulations from the binding free energy of the protein to the basal plane of ice, $\Delta G_{\text {bind }}=-57 \mathrm{~kJ} \mathrm{~mol}^{-1}$, 3 assuming that $\tau=10 \mathrm{pN}$ and its ice-binding area is $2.6 \mathrm{~nm}^{2}$, and then scaling $\Delta \gamma_{\text {bind }}=-61.8 \mathrm{~mJ} \mathrm{~m}^{-2}$ by $\gamma_{\text {ice-liquid }}($ water $) / \gamma_{\text {ice-liquid }}(\mathrm{mW})=$ $31.2 / 35$, as the value of $\gamma_{\text {ice-iquid }}$ modulates the maximum $T_{\text {het }}$ of an unlimited size surface. We use HINT with experimental data for water to determine $T_{\text {het }}{ }^{\text {unlim }}=264 \mathrm{~K}$ for the surface with $\Delta \gamma_{\text {bind }}=-55.1 \mathrm{~mJ} \mathrm{~m}^{-2}$ and $\tau=10 \mathrm{pN}$ at the nucleation rate $\omega=10^{2} \mathrm{~s}^{-1}$.

In both cases, the evaluate $T_{\text {het }}$ at the nucleation rate $\omega=10^{2} \mathrm{~s}^{-1}$ that leads to $T_{\text {hom }}=238 \mathrm{~K}$ in $\mu \mathrm{L}$ droplets (i.e. nucleation rate coefficient $J=10^{5} \mathrm{~cm}^{-3} \mathrm{~s}^{-1}$ ). As the area of the 34-mer of PsINP has an area that is $\sim 1000$ times that of TmAFP, and our reference for the calculation of $T_{\text {het }}$ of the proteins in experiment are droplets with $10^{4} \mathrm{Ps}$ syringae, or which only few are expected to be able to nucleate ice at $-2^{\circ} \mathrm{C},{ }^{6}$ we assign $N_{\mathrm{o}}$ here to a range of $10^{3}$ to $10^{7} \mathrm{TmAFP}$ per droplet. 


\section{E.1.1. Effect of concentration of TmAFP on $T_{\text {het }}$}

Table S3. Effect of number $N$ of TmAFP per droplet on its heterogeneous nucleation temperature. The results for the reference number of proteins per droplet, $N_{o}$ that ranges $10^{3}$ to $10^{7}$, are shown with bold font. The calculations are performed with HINT using the parameters of water and the nucleation rate that produces $T_{\text {hom }}=238 \mathrm{~K}$ for $\mu \mathrm{L}$ droplets.

\begin{tabular}{|c|c|c|c|}
\hline \multicolumn{4}{|c|}{$\Delta \gamma_{\text {bind }}=-62.6 \mathrm{~mJ} \mathrm{~m}{ }^{2}$ and $\tau=10 \mathrm{pN}$} \\
\hline$T_{\text {het }}(\mathrm{K})$ & $\ln \left(A\left(T_{\text {het }}\right)\right)$ & $\Delta G^{*}\left(T_{\text {het }}\right) / R T$ & $N / N_{0}$ \\
\hline 241 & $\mathbf{7 9 . 3}$ & $\mathbf{5 5 . 4}$ & $\mathbf{1}$ \\
\hline 242 & 79.5 & 60.2 & $10^{2}$ \\
\hline 243 & 79.8 & 66.5 & $10^{5}$ \\
\hline 244 & 80.1 & 73.4 & $10^{8}$ \\
\hline \multicolumn{4}{|c|}{$\Delta \gamma_{\text {bind }}=-55.1 \mathrm{~mJ} \mathrm{~m}^{2}$ and $\tau=10 \mathrm{pN}$} \\
\hline$T_{\text {het }}(\mathrm{K})$ & $\ln \left(A\left(T_{\text {het }}\right)\right)$ & $\Delta G^{*}\left(T_{\text {het }}\right) / R T$ & $N / N_{0}$ \\
\hline 240 & $\mathbf{7 9 . 0}$ & $\mathbf{5 5 . 1}$ & $\mathbf{1}$ \\
\hline 241 & 79.3 & 59.6 & 90 \\
\hline 242 & 79.5 & 65.3 & $10^{4}$ \\
\hline 243 & 79.8 & 71.6 & $10^{7}$ \\
\hline
\end{tabular}

\section{E.1.2. Effect on $T_{\text {het }}$ of aggregation of TmAFP.}

Table S4 presents the heterogeneous ice nucleation temperature predicted with HINT for TmAFP and maximum $T_{\text {het }}$ for the dimer and the trimer The HINT calculation assumes that the model is 2 $\mathrm{nm}$ long and $1.3 \mathrm{~nm}$ wide, the dimer is $2 \mathrm{~nm}$ long and $2.6 \mathrm{~nm}$ wide, the trimer is $2 \mathrm{~nm}$ long and 3.9 $\mathrm{nm}$ wide. The calculations in Table S4 assume that TmAFP dimers and trimers are flat and at distances that produce a good matching to ice. Hence, they are probably an upper limit to the $T_{\text {het }}$ of dimers and trimers of TmAFP in solution. The calculations assume that the binding efficiency of all these proteins and assemblies are the same as those deduced from experiments of $P S I N P(\tau=10 \mathrm{pN}$ and $\Delta \gamma_{\text {bind }}=-62.6 \mathrm{~mJ} \mathrm{~m}^{-2}$ at $272 \mathrm{~K}$ ) or those obtained from the binding free energy of TmAFP to the basal plane in the simulations of ref. ${ }^{3}$, scaled by the ratio of the ice-liquid surface tensions in the experiment and the $\mathrm{mW}$ water model at $273 \mathrm{~K}\left(\Delta \gamma_{\text {bind }}=-55.1 \mathrm{~mJ} \mathrm{~m}^{2}\right.$ and $\left.\tau=10 \mathrm{pN}\right)$. All calculations assume the same number of proteins per droplet, $N_{\mathrm{o}}$ between $10^{3}$ to $10^{7}$, of Table S3. 
Table S4. $T_{\text {het }}$ predicted for monomer and aggregates of TmAFP using HINT with the parameters for water and the two indicated sets of ice-binding properties of the protein surfaces.

\begin{tabular}{|c|c|c|c|c|c|c|}
\hline & \multicolumn{3}{|c|}{$\Delta \gamma_{\text {bind }}=-62.6 \mathrm{~mJ} \mathrm{~m}^{2}$ and $\tau=10 \mathrm{pN}$} & \multicolumn{3}{c|}{$\Delta \gamma_{\text {bind }}=-55.1 \mathrm{~mJ} \mathrm{~m}^{2}$ and $\tau=10 \mathrm{pN}$} \\
\hline$T$ mAFP & $T_{\text {het }}(\mathrm{K})$ & $\underline{\ln \left(A\left(T_{\text {het }}\right)\right)}$ & $\Delta G^{*}\left(T_{\text {het }}\right) / R T$ & $T_{\text {het }}(\mathrm{K})$ & $\underline{\ln \left(A\left(T_{\text {het }}\right)\right)}$ & $\Delta G^{*}\left(T_{\text {het }}\right) / R T$ \\
\hline monomer & 241 & 79.3 & 55.4 & 240 & 79.0 & 55.1 \\
\hline dimer & 245 & 80.3 & 56.5 & 244 & 80.1 & 56.2 \\
\hline trimer & 247 & 80.8 & 56.9 & 245 & 80.3 & 56.5 \\
\hline
\end{tabular}

\section{E.1.3. Effect on $T_{\text {het }}$ of lengthening the ice-binding-surface of TmAFP.}

Figure S4 shows the CNT predictions for the effect of lengthening the IBS of TmAFP by adding icebinding loops on $T_{\text {het, }}$ assuming that the ice-binding site is $1.3 \mathrm{~nm}$ wide and interacts with ice according to the two sets of surface-binding strengths indicated above. The calculations use the parameterization of HINT with the properties of water at experimental nucleation rates that result in $T_{\text {hom }}=238 \mathrm{~K}$. The increase in ice nucleation efficiency is severely limited by the narrow width of the ice-binding site of the antifreeze protein. This can be appreciated in the comparison of the blue curve of Figure S3, which reaches a maximum $\Delta T_{\mathrm{f}}=4 \mathrm{~K}$, and the one in Figure 6 for the $1.8 \mathrm{~nm}$ wide INP of $P$ s. syringae, which reaches a maximum $\Delta T_{\mathrm{f}}=9 \mathrm{~K}$.

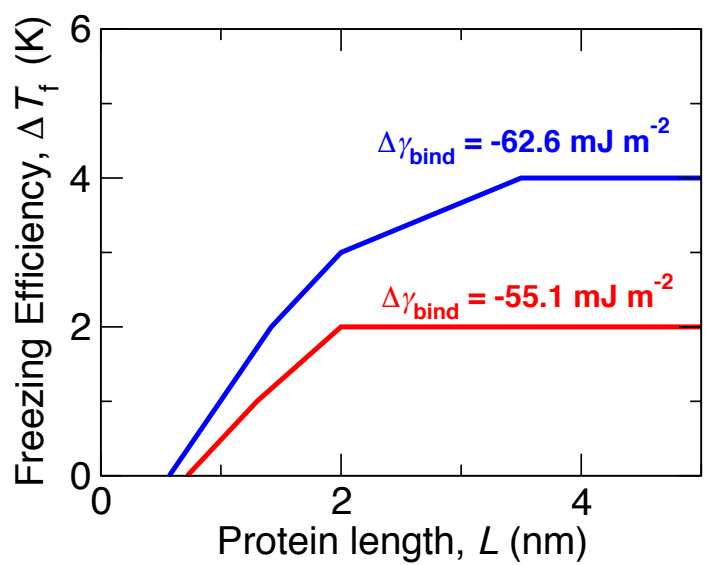

Figure S4. Effect of lengthening of the IBS on the ice nucleation efficiency of a protein with IBS 1.3 $\mathrm{nm}$ wide, considering two strengths of ice-protein interactions. The calculations are performed with $\tau=10 \mathrm{pN}$ and using the HINT parameterization for water at the rate that produces $T_{\text {hom }}=238 \mathrm{~K}$. The small gains in ice nucleation efficiency with length compared to TmINP are due to the different time scales of the experimental measurements compared to the simulation ones, see section E.1.4.

\section{E.1.4. Effect of the ice nucleation rate (observation time) on $T_{\text {het }}$}

Figure S5 illustrates the effect of the nucleation rate (observation time) on the measured freezing efficiency $\Delta T_{\mathrm{f}}$. The calculations in the figure are performed for the model TmINP as a function of the length of the monomer, using the parameterization of HINT for $\mathrm{mW}$ water. The results are qualitatively the same if the water parameterization is used. The figure shows that the slower the 
nucleation rate (the longer the observation times), the higher is the absolute $T_{\text {het }}$, but the smaller the raise of $T_{\text {het }}$ over $T_{\text {hom. }}$. $T_{\text {het }}$ for a given surface increases with as the nucleation rate coefficient $J$ decreases, because longer observation times allow for the formation of the more rare larger nuclei that are required at higher temperatures. The larger the nuclei, however, the least stabilization they receive from this narrow surface, resulting in lower $\Delta T_{\mathrm{f}}$.

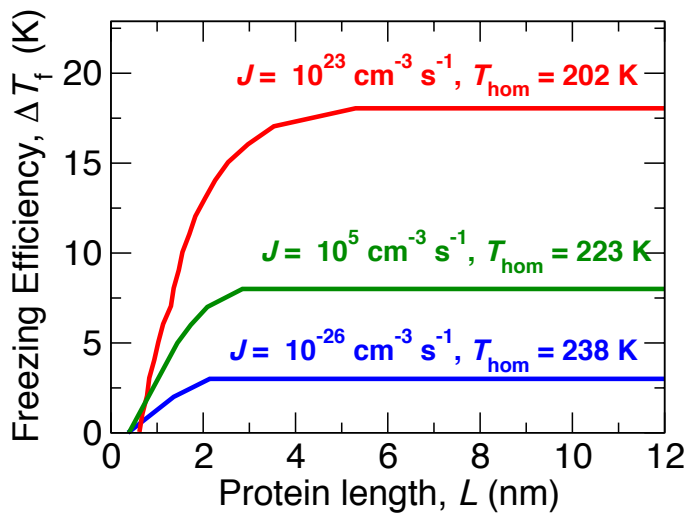

Figure S5. Freezing efficiency of the model TmINP as a function of length L for three distinct nucleation rate coefficients $\mathrm{J}$, which result in different Thom for the $\mathrm{mW}$ model. The nucleation temperatures were computed with the HINT algorithm using data for $\mathrm{mW}$ water and assuming the protein IBS has a width $1.3 \mathrm{~nm}, \Delta \gamma_{\text {bind }}=-68.1 \mathrm{mJm}^{-2}$ at $250 \mathrm{~K}, \tau=10 \mathrm{pN}$.

\section{E.2. Effect of concentration and aggregation on ice nucleation by PsINP}

Table S5 shows that the $T_{\text {het }}$ predicted for PsINP 34-mer is not sensitive to the amount of bacteria with 34-mer aggregates per droplet. The $T_{\text {het }}$ are computed with HINT using the geometry of the 34mer of PsINP ( $40 \mathrm{~nm}$ long by $61.2 \mathrm{~nm}$ wide) and the ice-binding thermodynamics we deduced for PsINP from the experimental data: $\tau=10 \mathrm{pN}$ and $\Delta \gamma_{\text {bind }}=-62.6 \mathrm{~mJ} \mathrm{~m}^{-2}$ at $272 \mathrm{~K}$, and nucleation rate is $\omega=10^{2} \mathrm{~s}^{-1}$ that leads to $T_{\text {hom }}=238 \mathrm{~K}$ in $\mu \mathrm{L}$ droplets. The reference experiment from which the $T_{\text {het }}$ $=271 \mathrm{~K}$ reported $10^{4}$ bacteria per $10 \mathrm{~mL}$ droplet, of which the authors conclude that just a few are able to nucleate ice at these high temperatures. ${ }^{6}$ Hence, the reference concentration of nucleating aggregate $N_{\mathrm{o}}$ can be considered to be at least 1 and at most $10^{4}$.

Table S5. Effect of number of bacteria per droplet with 34-mer PsINP on the heterogeneous nucleation temperature is negligible. The results for the reference concentration are shown with bold font.

\begin{tabular}{|c|c|c|c|}
\hline$T_{\text {het }}(\mathrm{K})$ & $\ln \left(A\left(T_{\text {het }}\right)\right)$ & $\Delta G^{*}\left(T_{\text {het }}\right) / R T$ & $N / N_{0}$ \\
\hline 270 & 84.8 & No barrier & $\begin{array}{c}\text { Any amount of this protein } \\
\text { aggregate nucleate ice. }\end{array}$ \\
\hline 271 & 85.0 & 61 & 1 \\
\hline 272 & 85.1 & $>5000$ & $\begin{array}{c}\text { Nucleation is impossible at this T } \\
\text { for any amount of this protein } \\
\text { aggregate; a larger one is required }\end{array}$ \\
\hline
\end{tabular}


Table S6. Effect of number of 16-loop long PsINP per droplet on the heterogeneous nucleation temperature. This protein is $8 \mathrm{~nm}$ long and $1.8 \mathrm{~nm}$ wide; the other parameters are same as in the calculations of Table S3. The results for the reference concentration are shown with bold font.

\begin{tabular}{|c|c|c|c|}
\hline$T_{\text {het }}(\mathrm{K})$ & $\ln \left(A\left(T_{\text {het }}\right)\right)$ & $\Delta G^{*}\left(T_{\text {het }}\right) / R T$ & $N / N_{0}$ \\
\hline 246 & 80.6 & 48 & $10^{-4}$ \\
\hline $\mathbf{2 4 7}$ & $\mathbf{8 0 . 8}$ & $\mathbf{5 7}$ & $\mathbf{1}$ \\
\hline 248 & 81.0 & 71 & $10^{6}$ \\
\hline 249 & 81.2 & 84 & $5 \times 10^{11}$ \\
\hline
\end{tabular}

Table S7. Maximum nucleation temperature $T_{\text {sat }}$ predicted with HINT for the aggregates of $P s I N P$ with binding free energy deduced from the maximum freezing temperature of Ps. syringae in experiments assuming that each monomer contributes $1.8 \mathrm{~nm}$ to the width and that their length is 40 $\mathrm{nm}$.

\begin{tabular}{|c|c|c|}
\hline $\begin{array}{c}\text { Number of } \\
P S I N P \\
\text { monomers }\end{array}$ & $\begin{array}{c}\text { Width of } \\
\text { IBS (nm) }\end{array}$ & $\begin{array}{c}T_{\text {sat }} \\
(\mathrm{K})\end{array}$ \\
\hline 1 & 1.8 & 247 \\
\hline 2 & 3.6 & 259 \\
\hline 3 & 5.4 & 263 \\
\hline 4 & 7.2 & 265 \\
\hline 5 & 9.0 & 266.5 \\
\hline 6 & 10.8 & 267.5 \\
\hline 8 & 14.4 & 268.5 \\
\hline 10 & 18.0 & 269 \\
\hline 16 & 28.8 & 270 \\
\hline 34 & 61.2 & 271 \\
\hline
\end{tabular}

\section{F. Configurations of Dimer of AlcoholINP.}

a)

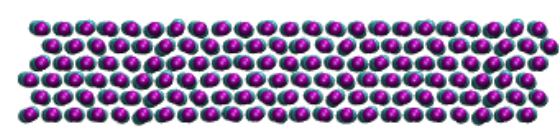

b)

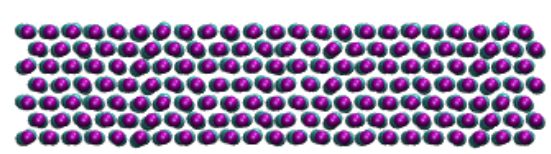

Figure S6. the snapshots of AlcohollNP dimers a) with one monomer shifted and b) dimers at peak II with the gap in the dimer filled with an extra column of binding sites. Color code is the same as Figure 1. Carbon tails are in cyan and hydroxyl groups are in purple. 


\section{References.}

(1) Qiu, Y.; Odendahl, N.; Hudait, A.; Mason, R. H.; Bertram, A. K.; Paesani, F.; DeMott, P. J.; Molinero, V., Ice Nucleation Efficiency of Hydroxylated Organic Surfaces Is Controlled by Their Structural Fluctuations and Mismatch to Ice. J. Am. Chem. Soc. 2017, 139, 3052-3064

(2) Hudait, A.; Moberg, D. R.; Qiu, Y.; Odendahl, N.; Paesani, F.; Molinero, V., Preordering of Water Is Not Needed for Ice Recognition by Hyperactive Antifreeze Proteins. Proc Natl Acad Sci USA 2018, 115, 8266-8271.

(3) Hudait, A.; Odendahl, N.; Qiu, Y.; Paesani, F.; Molinero, V., Ice-Nucleating and Antifreeze Proteins Recognize Ice through a Diversity of Anchored Clathrate and Ice-Like Motifs. J. Am. Chem. Soc. 2018, 140, 4905-4912.

(4) Moore, E. B.; Molinero, V., Structural Transformation in Supercooled Water Controls the Crystallization Rate of Ice. Nature 2011, 479, 506-508.

(5) Lupi, L.; Hudait, A.; Molinero, V., Heterogeneous Nucleation of Ice on Carbon Surfaces. J. Am. Chem. Soc. 2014, 136, 3156-3164.

(6) R. Maki, L.; Galyan, E. L.; Chang-Chien, M.-M.; Caldwell, D. R., Ice Nucleation Induced by Pseudomonas Syringae. Appl. Microbiol 1974, 28, 456-459.

(7) Lindow, S.; Lahue, E.; Govindarajan, A.; Panopoulos, N.; Gies, D., Localization of Ice Nucleation Activity and the Icec Gene Product in Pseudomonas Syringae and Escherichia Coli. Mol. Plant-Microbe Interact 1989, 2, 262-272.

(8) Eickhoff, L.; Dreischmeier, K.; Zipori, A.; Sirotinskaya, V.; Adar, C.; Reicher, N.; Braslavsky, I.; Rudich, Y.; Koop, T., Contrasting Behavior of Antifreeze Proteins: Ice Growth Inhibitors and Ice Nucleation Promotors. J. Phys. Chem. Lett. 2019, 10, 966-972. 